\title{
Early intervention in psychosis: strengths and limitations of services ${ }^{\dagger}$
}

\author{
Brendan P. Murphy \& Warrick J. Brewer
}

\begin{abstract}
SUMMARY
Early intervention services were established on the basis of a number of fundamental principles, including the notions that intervening in the early stages of psychosis alters illness trajectory and prognosis, that multicomponent interventions promote psychosocial recovery and reduce iatrogenic damage, and that early targeting of non-responders reduces treatment resistance. There is growing evidence of the benefits of specialised early intervention services. These include improved clinical, social and vocational outcomes, reduced in-patient stays and better engagement. Early intervention services can also significantly reduce the risk of a second episode and are highly valued by service users and carers. Duration of treatment appears to determine longterm outcome and there remains uncertainty about how long such intensive intervention should last and whether all patients need the same length of care. Budgetary constraints are pervasive and are particularly likely to affect prodrome clinics and community awareness programmes.
\end{abstract}

\section{DECLARATION OF INTEREST}

B.P.M. has received research support from Diabetes Australia, the Australian Heart Foundation, Eli Lilly and Sanofi; participated in clinical trials sponsored by Sanofi, Eli Lilly, AstraZeneca, Jannsen-Cilag and Lundbeck; and been a speaker for Bristol-Myers Squibb, Lundbeck and Pfizer. W.J.B. is supported by a National Health and Medical Research Council Career Development Award and the Colonial Foundation, and has received research support from Janssen-Cilag and Eli Lilly.

First-episode psychoses, including schizophrenia, are currently best understood as neurodevelopmental illnesses arising from the interaction of assorted biological and psychosocial factors. Although an excess loading of genetic susceptibility and environmental risk factors may manifest in childhood through a range of non-specific, subtle behavioural changes, the vulnerability is usually silent until the onset of prodromal or psychotic symptoms in the mid-teens to early 20s (Welham
2009). Early intervention services were established on the basis of a number of fundamental principles. These include the notions (McGlashan 1996; McGorry 1996) that:

- intervention in the early stages of psychosis can change the trajectory of illness and ameliorate long-term prognosis;

- multicomponent intervention promotes psychosocial recovery, and reduces iatrogenic damage and carer burden;

- early targeting of non-responders can reduce treatment resistance.

The concept of the 'critical period' (Birchwood 1998 ) - the first 3-5 years from psychosis onset, which in most people coincide with maximal neuronal and psychosocial plasticity and when, theoretically, the greatest impact on illness can be made - facilitated extension of the approach to include 'indicated prevention' in the prodromal stage (Yung 2003). In indicated prevention, special intervention programmes are targeted at individuals who are showing early signs and other related problem behaviours. The rationale was complemented by the assumptions of a clinical staging model proposing that treatment in the early rather than late stages of illness is more benign and results in better response and prognosis, thus minimising the need for traumatic and restrictive forms of care, including involuntary hospital admissions (McGorry 2006, 2010a).

Melbourne, Australia, has a strong case for being considered the cradle of early intervention, with the pioneering Early Psychosis Prevention and Intervention Centre (EPPIC) established in 1992 (McGorry 1996). The service, currently known as Orygen Youth Health Research Centre, subsequently expanded to provide intervention for all youth mental health problems. More recently, it drove the development of the 'headspace' consortium, a federally funded national youth mental health initiative to promote and support early intervention for young people with mental and substance use disorders (McGorry 2007). There has been rapid expansion of early
Brendan Murphy is a consultant psychiatrist and Clinical Director of the Recovery and Prevention of Psychosis Service in Melbourne and Associate Professor at Monash University, Australia He has established a research programme investigating medical comorbidity, negative symptoms, novel treatments and genetic epidemiology of psychosis, and has an interest in neurotrophins and the autoimmune aetiology of the disorder. He is currently developing a fully integrated youth service. Warrick Brewer is Associate Professor and Principal Research Fellow in Neuropsychology at Orygen Youth Health Research Centre, Neuropsychology Research Unit, University of Melbourne. $\mathrm{He}$ is also the specialist clinical consultant for the EPPIC Intensive Case Management subprogramme. His primary research interests include using olfactory measures to identify neurodevelopmental risk of pathology, with an emphasis on psychosis and antisocial personality disorder. Correspondence Associate Professor Brendan P. Murphy, Early in Life Mental Health Service, Community Services Building, 145 Cleeland Street, Dandenong, Victoria, Australia, 3175. Email: brendan.murphy@ monash.edu

${ }^{\dagger}$ For a companion article and related Editorial see pp. 408-16 and 398-400, this issue. 
intervention services in many regions, notably Australia, Canada, Scandinavia and the UK. In England alone, 118 services are now operating (Tiffin 2007).

It is important to note that 'early intervention' may refer to one of three service aims, all of which require different strategies (Singh 2010). It can mean improving outcomes in established cases of psychosis; or having an early detection component (for example, through community awareness campaigns and primary care education) to identify hidden morbidity, with the intention of reducing the mean duration of untreated psychosis (DUP) in the service's cohort; or preventing the emergence of psychosis in individuals with prodromal symptoms through the establishment of prodrome clinics for help-seeking patients (Singh 2007). The presence of prodrome clinics and community awareness campaigns varies considerably, depending both on resources and embedded research endeavours. Although there is emerging evidence of the value of these components, they are often threatened by lack of finances. It is essential that service planners safeguard consistent funding to reflect these broader aims.

\section{Strengths and limitations of variations in service models}

Although early intervention services generally follow standardised principles of care (Box 1), they may vary in their model of service delivery (Box 2). Each variation has its strengths and weaknesses, and the model adopted in any particular service is often influenced primarily by feasibility and economies of scale rather than ideological frameworks.

\section{Age range}

Although age ranges vary, services are generally divided into those with a low age cut-off (typically

BOX 1 Key principles of service provision in early psychosis

- Assertive community treatment model
to facilitate the early identification and
management of those with first-episode
psychosis
- Youth-friendly in-patient and out-patient
services
- Multidisciplinary, phase-oriented
treatment coordinated by a case manager
- Focus on early detection, recovery and
relapse prevention
- Low-dose antipsychotic medication

- Targeted psychological interventions

- Management of secondary comorbid conditions, including affective and substance use disorders

- Engagement of families, including the provision of psychoeducation

- Psychosocial recovery focus, including educational, vocational and residential support, with the aim of maintaining community engagement

\section{BOX 2 Differences in service configuration}

- Age range

- Case-load

- Duration of care

- Embedded $v$. stand-alone

- Centralised $v$. hub-and-spoke

- Hours of business

- Inclusion of research programmes

- Inclusion of specialised components

- Provision of health promotion and community awareness

- Provision of physical health monitoring

24 or 25 years of age), to maintain a youth focus, and those with a higher cut-off (30 or even 40 years of age), to include as many people with early psychosis as possible. The principal advantage of a youth-focused service is that it provides developmentally appropriate interventions to the majority of those with a first-episode psychosis. The disadvantages are twofold.

First, youth-focused services generally have a cutoff at age 25, and anyone older is treated by generic adult mental health services. This is a significant issue, as a substantial cohort develops psychosis between 25 and 35 years of age, especially women and people with bipolar disorder. Such patients are then treated in generic services, which are universally characterised by long delays in treating first-episode psychosis (Norman 2001; Farooq 2009) as well as by iatrogenic damage (see the subsection on in-patient units later in this article), which leads to high rates of disengagement (Garety 2001; McGorry 2010a). Rather than compromising a youth-specific model at the expense of including the maximum number of people with first-episode psychosis, it may be better to compel the argument for promoting service reform in adult mental health by developing and resourcing similar models of early intervention across the age range.

Second, youth-focused services by definition treat those under 18 as well as those up to the mid-20s age cut-off, thus disrupting the traditional demarcation between child and adult psychiatry. This has resulted in debate as to whether early intervention services (and youth mental health services generally) are best managed by child and adolescent mental health services, adult mental health services or whether they should constitute separate entities (Birleson 2008; McGorry 2008). Although the development of youth-specific services appears intuitive and has an emerging evidence base in support of service 
reform (McGorry 2007), there appears to be no definitive answer as to where they are best placed. The local solution in Victoria, Australia, has been to introduce health reform aimed at facilitating dedicated service streams planned and managed across a broad 0-25 years framework (Department of Health 2009).

\section{Case-loads}

Most early intervention services were established with fixed case-loads of 15 patients. However, many have found that service delivery has been compromised by budgetary constraints, resource realignment that lags behind demand, and delays in recruitment and infrastructure establishment (Lester 2009; Murphy 2009). This often results in higher case-loads, compromising holistic early intervention treatment and/or more focused, evidence-based treatment of specific comorbid conditions such as post-traumatic stress disorder (PTSD). In the UK, where meeting target caseloads of 15 is linked to ongoing funding, case-loads tend to be maintained at the expense of other survival strategies, such as early discharge, age-range restriction, imposing waiting lists and reducing community development work (Lester 2009).

In Australia, some services (EPPIC, for example) were originally set up with higher case-loads (McGorry 1996) or have introduced the concept of 'step-down' care to cater for individuals who are either subject to a shared care arrangement or need minimal contact, allowing increased caseloads without compromising patient care (Murphy 2009).

Finally, there is increasing recognition of the need to develop ultra-intensive case management teams, with case-loads of 10 , for patients with highest or unmet needs (Crlenjak 2008).

\section{Duration of care}

Services vary in the tenure of care offered to patients, but the usual duration is $2-3$ years. In the UK, early intervention services were established to provide a 3-year fixed term, whereas early intervention services in Australia provide up to 3 years, titrating length of care against need. There is increasing recognition that $2-3$ years is not adequate for a significant minority of people with first-episode psychosis and some services now provide up to 5 years' care for selected patients, to cover the critical period (Murphy 2009).

\section{Stand-alone v. embedded services}

Early intervention services may either be standalone, where they work only with first-episode psychosis, or embedded, where they are part of community mental health teams. A key benefit of the embedded model is the potential for more seamless continuity and shared responsibility for the early intervention cohort; the key disadvantage is the potential for dilution of the early intervention philosophy (Murphy 2009). The adopted model is often influenced by feasibility and economies of scale, rather than particular ideological frameworks. For example, healthcare providers with large catchment areas may have sufficient patients to justify a stand-alone early intervention service, whereas those serving smaller populations may not. Similarly, the ability to introduce a huband-spoke model, where services develop satellite clinics in a bid to tailor themselves to the needs of the community, may be a function of size.

\section{Research programmes}

The establishment of early intervention services has enabled crucial evidence to be amassed (McGorry 2010a). Hybrid clinical-research programmes hold key advantages (Good 2004). Supplementary research resources can lead to enhanced and more comprehensive care, with patients, carers and staff having ready access to cutting-edge evidence. Evidence-based practice can be more easily assessed in a clinical setting, and the embedding of research and clinical frameworks in a single service enables ongoing, efficient evaluation of the work of both. Furthermore, such a unit can attract leading international researchers and clinicians.

It is important that embedded research resources do not become an exploitative rationale for reduced public funding of early intervention services. Similarly, the misperception that scarce clinical resources are being diverted to support research requires containment. Such polarising perspectives serve only to dilute the advantages pointed out by Good et al (2004).

\section{Specialised components}

Comprehensive early intervention services ideally incorporate a number of additional components such as those listed in Box 3.

B0X 3 Additional service components

- A prodrome clinic

- Community and referrer education and awareness campaigns

- Dedicated in-patient services

- A crisis team

- Specialised subprogrammes such as group programmes

- Physical health monitoring 


\section{Prodrome clinics}

Prodrome clinics target help-seeking individuals identified as likely to be at incipient risk of developing psychosis. Their key advantage is that those who meet intake criteria have a better chance of responding to the benefits of early intervention, such as ameliorating the severity of, and extent of trajectory into, crystallised illness. A further advantage is that prodrome clinics also identify cohorts of patients at risk of developing non-psychotic disorders. The challenge here is to differentiate presentations, as the early stages of both psychotic and non-psychotic disorders have overlapping phenotypes (McGorry 2010b). Prodrome clinics, then, are ideally positioned to further refine our understanding of illness genesis across the diagnostic spectrum.

A key disadvantage is that prodrome clinics fail to cater for the majority of those with first-episode psychosis, as these individuals generally do not seek help. This suggests a need to review intake criteria and increase public awareness. Other problems include an excess of false positives, which leads to the risk that early intervention services will become overburdened, and the issue of treating individuals at ultra-high risk of psychosis; even in the presence of psychosis-like symptoms, only $40-60 \%$ progress to psychosis (van Os 2009). This triggers anxieties and strong debate over whether to treat prodromal symptoms with antipsychotics. Certainly, methods to improve the detection of true cases, including the determining and measuring of premorbid risk factors, need continual refinement (Murphy 2010).

Current international clinical guidelines are conservative and suggest a generalist approach to treatment during the prodrome, with monitoring and psychosocial interventions (International Early Psychosis Association Writing Group 2005). Positive results have been reported for a number of intervention studies. These include randomised controlled trials (RCTs) of cognitive-behavioural therapy (CBT), low-dose risperidone and essential fatty acids, and open-label trials of antidepressants, aripiprazole and the amino acid glycine (for review see McGorry 2010b). Olanzapine was not found to be beneficial (McGlashan 2006).

Early prodromal symptoms - negative symptoms, anxiety and depression - can merge imperceptibly with attenuated psychotic symptoms until precipitation into frank psychosis occurs. Initiation of antipsychotics in the early prodrome may be unhelpful, since these early symptoms are largely refractory to antipsychotic medication (Murphy 2006). Symptom-specific treatments such as antidepressants and CBT are likely to be of more benefit. They also serve to minimise iatrogenesis arising from the 'treatment' of false positives. In true cases, it is the onset of attenuated psychotic symptoms that is likely to represent the start of the individual's psychosis, not the diagnostic classificatory requirements for established symptoms of prolonged duration (World Health Organization 1992; American Psychiatric Association 1994). It is not until such symptoms begin that antipsychotics are likely to be beneficial, although initiation at this stage can significantly improve the prognosis. Differentiating true cases from false positives (with psychotic-like or spontaneously resolving symptoms), however, continues to be problematic.

\section{In-patient units}

A focus on early intervention minimises the need for later traumatic and restrictive forms of care, including involuntary hospital admission (McGorry 2010a). Nevertheless, the majority of people with first-episode psychosis require at least one in-patient admission (Wade 2006). For these individuals, it is important to reduce iatrogenicity by avoiding exposure to adult wards, with their high rates of seclusion, chronically ill older patients and overreliance on rapidly titrated highdose antipsychotics (Murphy 2009). In-patient care should be provided either in specialised early psychosis units that are age and developmentally appropriate or on a youth ward. Wards should be small in bed numbers and have enough staff to prevent locking of the unit (Bertolote 2005).

\section{Subprogramme development}

A significant effect of focusing specialised services on first-episode cohorts has been an increased understanding of the variable trajectories of illness progression. The original 'one size fits all' model of early intervention services has revealed subgroups of patients with unmet need. These include people with premorbid cognitive risk factors, dissociative borderline personality features, antisocial and/or drug-induced psychosis, and treatment resistance, all of which are conditions that benefit from bespoke treatment initiatives. Articulation of inclusion criteria inherently serves to identify and characterise subgroups, and leads to a more practical focus for research and future funding.

Specialised formal subprogrammes mightinclude neuropsychology and intensive case management for patients at high risk of homicide, suicide and/or disengagement, treatment resistance or persisting negative symptoms, comorbid autism-spectrum disorders, and psychosis with a predominantly Axis II and/or substance-induced foundation. 


\section{Effectiveness of early intervention}

It is difficult to appraise and synthesise the literature on effectiveness of early intervention in psychosis: the complexity of multicomponent intervention means it is unlikely that specialised teams are identical, making comparisons difficult (Marshall 2006). This is reflected in the Cochrane Database findings, which quantitatively reviewed the efficacy of RCTs of early intervention. Of the 65 studies identified, 58 were excluded for methodological reasons, resulting in inconclusive findings (Marshall 2006). Nevertheless, there is gathering evidence of the benefits of specialised early intervention services across a range of study designs (Catts 2010). Indeed, there is more evidence supporting early intervention in psychosis than all other recent mental healthcare service initiatives, including assertive community treatment and home treatment services (Lester 2009).

Several RCTs in the UK, Norway and Denmark have demonstrated various beneficial results, including improved clinical, social and vocational outcomes, reduced in-patient stays and better engagement (Singh 2010). Early intervention services have also been found to significantly reduce the risk of a second episode (AlvarezJiménez 2011) and are highly valued by service users and carers (Lester 2009). Identifying and treating patients with a shorter DUP results in less severe symptoms, reduced suicidality (Melle 2004; Larsen 2006) and better functional and social outcomes at 2 years (Melle 2008; Larsen 2011). Patients with a longer DUP, and consequently an expected poorer prognosis, are also engaged and treated earlier and more effectively in early intervention than in generic services (Friis 2005), supporting the argument that even the cohort with the most severe symptoms benefits from early specialised care. Providing general practitioners with education sessions and easy access to early intervention services increases referrals, but does not necessarily reduce DUP (Power 2007).

\section{Duration of treatment}

It does appear that length of tenure determines outcome in the longer term, and that gains made are lost when patients are discharged to generic services (Bertelsen 2009; Gafoor 2010; Henry 2010). Rather than indicating a failure of early intervention services, this is more likely to reflect an insufficient period of exposure to their therapeutic benefits. A study showing a well-functioning cohort on discharge from early intervention services who subsequently deteriorate would help clarify this. There remains uncertainty about how long such intensive early intervention should last and whether all patients need the same length of care (Singh 2010). Most services provide 2-3 years of care (Tiffin 2007). However, it appears intuitive to ensure intensive intervention to cover the 'critical period' for at least the more severely ill patients, and some services are now retaining selected patients for this duration (Murphy 2009).

Although it is clear that there is a humanitarian imperative to offer bespoke treatment at onset, the concept of the critical period itself presents a dilemma. Although there is evidence of neurobiological and neurocognitive changes prior to, and at, early onset, these are relatively unstable (Pantelis 2009). Recent evidence does indicate that neurobiological interventions are beneficial in ameliorating transition to psychosis, but further longitudinal research is required (McGorry 2009).

\section{Areas for further research}

Future research should attempt to clarify not only which patients are likely to benefit from extended care, but the key therapeutic ingredients of early intervention services that are effective. This, in turn, would inform on elements of maintenance care when patients are discharged to generic services (Singh 2010). There is no reason why early intervention initiatives cannot be subsumed within generic programmes, provided that the key therapeutic ingredients are resourced and maintained. This would, however, require a significant change in philosophy for generic service providers, who have traditionally failed to deliver, thus providing the rationale for development of early intervention services in the first place. It is a moot point whether any of the specialised subprogrammes or research findings would have been produced within a generic service structure.

Undoubtedly, healthy debate is to be encouraged, and those few within generic services who advocate for the delivery of sustained specialised intervention are to be applauded (Castle 2010), but a focus on resourcing clinical research frameworks that further characterise and refine these subgroups is likely to be a more productive way forward.

Finally, regarding cost-effectiveness, there is a growing, consistent body of evidence, all of which reports the economic benefits of early intervention services, mainly due to reduced bed-days (Catts 2010; Singh 2010).

\section{Extended care}

Determining which patients will need extended tenure (and which will not) is crucial for the continuing viability and effectiveness of early intervention services. Furthermore, early identification 
MCO answers

$\begin{array}{lllll}1 b & 2 d & 3 c & 4 e & 5 d\end{array}$ of schizophrenia in first-episode psychosis may help avoid inappropriate treatment and enhance long-term outcomes (Ramirez 2010).

\section{The DUP}

Although the meaning of 'duration of untreated psychosis' might seem clear - it is the period from onset of psychosis to the start of treatment - precise definitions vary widely in practice and there is no consensus over when it begins and, to a lesser degree, ends (Singh 2007). In early intervention services, the DUP is commonly used as a standalone predictor of outcome, despite its modest abilities in predicting recovery in both the short and medium term (Marshall 2005; Perkins 2005). There has been justifiable criticism of this usage (Bosanac 2010), but the overemphasis on DUP in the early intervention community may be related to its historical importance in justifying capital funding.

The use of the DUP as a stand-alone measure of prognosis has a number of limitations. First, it fails to capture the true 'pathogenic portrait' for many individuals with psychosis who have suffered significant premorbid adjustment problems. Second, it does not inform on the prodrome, which may extend for a number of years before the onset of psychosis. Finally, since the offset of the DUP coincides with treatment, it does not take into account the toxic effects of ongoing psychosis that affect many patients.

\section{Prognosis in schizophrenia}

There are a host of known and putative premorbid risk factors for schizophrenia that might add prognostic weight (for a review see Murphy 2010), yet are not routinely used. Among them is premorbid adjustment, which has a distinguished track record in prognosis (McGlashan 2008). Although there is currently no predictive model incorporating premorbid risk factors, it makes sense to map them clinically to gain a more accurate sense of which patients are likely to progress to schizophrenia and, of those that do, which are likely to have worse outcomes. In this mapping, the presence of known risk factors can be combined with DUP, clinical characteristics and other prognostic indicators including the postmorbid risk factors of ongoing positive, negative and cognitive symptoms, medication non-adherence and ongoing substance misuse (Murphy 2010). By engaging in this process, early intervention services should be able to better predict those who would benefit from continued treatment, titrating care against need.

In the second of our two articles in this issue (Murphy 2011), we discuss clinical aspects of early intervention.

\section{References}

Alvarez-Jiménez M, Parker AG, Hetrick SE, et al (2011) Preventing the second episode: a systematic review and meta-analysis of psychosocial and pharmacological trials in first-episode psychosis. Schizophrenia Bulletin 37: 619-30.

American Psychiatric Association (1994) Diagnostic and Statistical Manual of Mental Disorders (4th edn) (DSM-IV). APA.

Bertelsen M, Jeppesen P, Petersen L, et al (2009) Course of illness in a sample of 265 patients with first-episode psychosis: five-year follow-up of the Danish OPUS trial. Schizophrenia Research 107: 173-8.

Bertolote J, McGorry P (2005) Early intervention and recovery for young people with early psychosis: consensus statement. British Journal of Psychiatry 187 (suppl 48): s116-9.

Birchwood M, Todd P, Jackson C (1998) Early intervention in psychosis: the critical period hypothesis. British Journal of Psychiatry 172 (suppl 33): $553-9$

Birleson P, Vance A (2008) Developing the 'youth model' in mental health services. Australasian Psychiatry 16: 22-6.

Bosanac P, Patton GC, Castle DJ (2010) Early intervention in psychotic disorders: faith before facts? Psychological Medicine 40: 353-8.

Castle D, Bosanac P, Patton G (2010) Early intervention in psychosis: a response to McGorry et al (2010). Psychological Medicine 40: 2108-9.

Catts SV, O’Toole BI, Carr VJ, et al (2010) Appraising evidence for intervention effectiveness in early psychosis: conceptual framework and review of evaluation approaches. Australian and New Zealand Journal of Psychiatry 44: 195-219.

Crlenjak C, Gee D, Duff C, et al (2008) Engaging high suicide risk early psychosis clients via intensive case management. 6th International Conference on Early Psychosis, Melbourne. Early Intervention in Psychiatry 2 (suppl 1): A82.

Department of Health (2009) Because Mental Health Matters: Victorian Mental Health Reform Strategy 2009-2019. Department of Health, Victoria, Australia (www.health.vic.gov.au/mentalhealth/reformstrategy).

Farooq S, Large M, Nielssen 0, et al (2009) The relationship between the duration of untreated psychosis and outcome in low-and-middle income countries: a systematic review and meta analysis. Schizophrenia Research 109: 15-23.

Friis S, Vaglum P, Haahr U, et al (2005) Effect of an early detection programme on duration of untreated psychosis: part of the Scandinavian TIPS study. British Journal of Psychiatry 187 (suppl 48): s29-32.

Gafoor R, Nitsch D, McCrone P, et al (2010) Effect of early intervention on 5-year outcome in non-affective psychosis. British Journal of Psychiatry 196: 372-6.

Garety PA, Rigg A (2001) Early psychosis in the inner city: a survey to inform service planning. Social Psychiatry and Psychiatric Epidemiology 36: $537-44$.

Good KP, Brewer WJ, Kopola LC (2004) The benefits of incorporating research into clinical programs. In Best Care in Early Psychosis Intervention (eds T Ehmann, GW MacEwan, WG Honer): 175-82. Taylor \& Francis.

Henry LP, Amminger GP, Harris MG (2010) The EPPIC follow-up study of first-episode psychosis: longer-term clinical and functional outcome 7 years after index admission. Journal of Clinical Psychiatry 71: 716-28.

International Early Psychosis Association Writing Group (2005) International clinical practice guidelines for early psychosis. British Journal of Psychiatry 187 (suppl 48): s120-4.

Larsen TK, Melle I, Auestad B, et al (2006) Early detection of first-episode psychosis: the effect on 1-year outcome. Schizophrenia Bulletin 32: 758-64

Larsen TK, Melle I, Auestad B, et al (2011) Early detection of psychosis: positive effects on 5-year outcome. Psychological Medicine 41: 1461-9.

Lester H, Birchwood M, Bryan S, et al (2009) Development and implementation of early intervention services for young people with psychosis: case study. British Journal of Psychiatry 194: 446-50.

Marshall M, Lewis S, Lockwood A, et al (2005) Association between duration of untreated psychosis and outcome in cohorts of first-episode patients: a systematic review. Archives of General Psychiatry 62: 975-83. 
Marshall M, Rathbone J (2006) Early intervention for psychosis. Cochrane Database of Systematic Reviews issue 4: CD004718.

McGlashan T, Johannessen J0 (1996) Early detection and intervention in schizophrenia: rationale. Schizophrenia Bulletin 22: 201-22.

McGlashan T, Zipursky RB, Perkins D, et al (2006) Randomized, doubleblind trial of olanzapine versus placebo in patients prodromally symptomatic for psychosis. American Journal of Psychiatry 163: 790-9.

McGlashan TH (2008) Premorbid adjustment, onset types, and prognostic scaling: still informative? Schizophrenia Bulletin 34: 801-5.

McGorry PD, Edwards J, Mihalopoulos C, et al (1996) EPPIC: an evolving system of early detection and optimal management. Schizophrenia Bulletin 22: 305-26.

McGorry PD, Hickie IB, Yung AR, et al (2006) Clinical staging of psychiatric disorders: a heuristic framework for choosing earlier, safer and more effective interventions. Australian and New Zealand Journal of Psychiatry 40: 616-22.

McGorry PD, Tanti C, Stokes R, et al (2007) headspace: Australia's National Youth Mental Health Foundation - where young minds come first. Medical Journal of Australia 187 (suppl 7): s68-70.

McGorry PD, Hazell P, Hickie I, et al (2008) The 'youth' model in mental health services. Australasian Psychiatry 16: 136-7.

McGorry PD, Nelson B, Amminger GP, et al (2009) Intervention in individuals at ultra-high risk for psychosis: a review and future directions. Journal of Clinical Psychiatry 70: 1206-12.

McGorry PD, Johanessen J0, Lewis S, et al (2010a) Early intervention in psychosis: keeping faith with evidence-based health care. Psychological Medicine 40: 399-404.

McGorry PD, Nelson B, Goldstone S, et al (2010b) Clinical staging: a heuristic and practical strategy for new research and better health and social outcomes for psychotic and related mood disorders. Canadian Journal of Psychiatry 55: 486-97.

Melle I, Larsen TK, Haahr U, et al (2004) Reducing the duration of untreated first-episode psychosis: effects on clinical presentation. Archives of General Psychiatry 61: 143-50.

Melle I, Larsen TK, Haahr U, et al (2008) Prevention of negative symptom psychopathologies in first-episode schizophrenia: two-year effects of reducing the duration of untreated psychosis. Archives of General Psychiatry 65: 634-40.

Murphy BP, Chung Y-C, Park T-W, et al (2006) Pharmacological treatment of primary negative symptoms in schizophrenia: a systematic review. Schizophrenia Research 88: 5-25.

Murphy BP, Simms C, Dowling R-M, et al (2009) On giant's shoulders: the development of the Recovery and Prevention of Psychosis Service (RAPPS) in Melbourne, Australia. Early Intervention in Psychiatry 3: 94-9.
Murphy BP (2010) Beyond the first episode: candidate factors for a risk prediction model of schizophrenia. International Review of Psychiatry 22 : 202-23.

Murphy B, Brewer W (2011) Early intervention in psychosis: clinical aspects of treatment. Advances in Psychiatric Treatment 17: 408-16.

Norman RMG, Malla AK (2001) Duration of untreated psychosis: a critical examination of the concept and its importance. Psychological Medicine 31: $381-400$

Pantelis C, Yucel M, Bora E, et al (2009) Neurobiological markers of illness onset in psychosis and schizophrenia: the search for a moving target. Neuropsychological Review 19: 385-98.

Perkins DO, Gu H, Boteva K, et al (2005) Relationship between duration of untreated psychosis and outcome in first-episode schizophrenia: a critical review and meta-analysis. American Journal of Psychiatry 162 : 1785-804.

Power P, lacoponi E, Reynolds N, et al (2007) The Lambeth Early Onset Crisis Assessment Team Study: general practitioner education and access to an early detection team in first-episode psychosis. British Journal of Psychiatry 191 (suppl 51): s133-9.

Ramirez N, Arranz B, Salavert J, et al (2010) Predictors of schizophrenia in patients with a first episode of psychosis. Psychiatry Research 175: 11-4. Singh SP (2007) Outcome measures in early psychosis: relevance of duration of untreated psychosis. British Journal of Psychiatry 191 (suppl 50): s58-63.

Singh SP (2010) Early intervention in psychosis. British Journal of Psychiatry 196: 343-5.

Tiffin P, Glover G (2007) From commitment to reality: early intervention in psychosis services in England. Early Intervention in Psychiatry 1: 104-7.

van Os J, Linscott RJ, Myin-Germeys I, et al (2009) A systematic review and meta-analysis of the psychosis continuum: evidence for a psychosis proneness-persistence-impairment model of psychotic disorder. Psychological Medicine 39: 179-95.

Wade D, Harrigan S, Harris MG, et al (2006) Pattern and correlates of inpatient admission during the initial acute phase of first-episode psychosis. Australian and New Zealand Journal of Psychiatry 40: 429-36.

Welham J, Isohanni M, Jones P, et al (2009) The antecedents of schizophrenia: a review of birth cohort studies. Schizophrenia Bulletin 35 603-23.

World Health Organization (1992) The ICD-10 Classification of Diseases, Clinical Descriptions and Diagnostic Guidelines. WHO

Yung A, Phillips LJ, Yuen HP, et al (2003) Psychosis prediction: 12-month follow up of a high-risk ('prodromal') group. Schizophrenia Research 60 21-32.

\section{MCQs}

Select the single best option for each question stem

1 The city considered to be the cradle of early intervention in psychosis is:

a Vancouver

b Melbourne

c London

d Stockholm

e New York.

\section{Which of the following is not a key} principle of service provision?

a youth-friendly in-patient and out-patient services

b targeted psychological interventions

c focus on early detection, recovery and relapse prevention d in-patient admission to ensure comprehensive assessment of all first-episode cases

e low-dose antipsychotic medication.

3 Most early intervention services have
fixed case-loads of:
a 5
b 10
c 15
d 20
e 25 .

4 Which of the following is not a specialised component of early intervention services?

a a crisis team

b an in-patient unit

c a prodrome clinic d a community and referrer education and awareness programme

e a speech therapy clinic

5 The critical period refers to the following length of time from psychosis onset:

a 1 week

b 1 month

c 1 year

d $3-5$ years

e $5-10$ years. 